\title{
Effect of Miao medicine, Jinwujiangu decoction, on IL- $17 / I L-23$ inflammatory axis of fibroblast-like synoviocytes in rheumatoid arthritis
}

\author{
Xueming Yao, Rong Li, Qiaoyi Ning, Ying Huang, Fang Tang, Hui Xu, Daoming \\ Lu, Jiang Liang, Yang An, Wukai Ma* \\ Department of Rheumatology and Immunology, Second Affiliated Hospital of Guiyang College of Traditional Chinese Medicine, \\ Fei Shan Street, Guiyang 550003, China
}

*For correspondence: Email: mawukaituo@163.com; Tel: +86-0851-85556970

\begin{abstract}
Purpose: To explore the influence of the Miao medicine, Jinwujiangu decoction, on the interleukin (IL)17/L-23 inflammatory axis of fibroblast-like synoviocytes (FLS) in rheumatoid arthritis (RA).

Methods: Synovial tissue samples were randomly divided into a blank control group, high-dose $(0.06 \mathrm{mg} / \mathrm{mL})$, medium-dose $(0.6 \mathrm{mg} / \mathrm{mL})$, and low-dose $(6.0 \mathrm{mg} / \mathrm{mL})$ groups of Jinwujiangu decoction, a leflunomide group, and a tripterygium glycosides group. Proliferation of RA synovial cells was detected by 3-[4,5-dimethylthiazol-2-yl]-2,5 diphenyl tetrazolium bromide (MTT) assay. Enzyme-linked immunosorbent assay (ELISA) was used to determine the secretion of IL-6, transforming growth factor beta (TGF- $\beta$ ), and IL-17. Real-time polymerase chain reaction was used to evaluate the expression of IL-23R, IL-17R, RAR-related orphan receptor alpha (RORa), RORyt, and signal transducer and activator of transcription (STAT3) mRNA. The protein activities of IL-17R, STAT3 and pSTAT3 were assessed by Western blot assay.

Results: Jinwujiangu decoction inhibited the proliferation of RA synovial cells. Treatment with different drug concentrations resulted in downregulation of IL-6, TGF- $\beta$, and IL-17 secretion. The expression levels of IL-23R, IL-17R, RORa, RORyt, and STAT3 mRNA in RA-FLS were significantly reduced after intervention with different drugs. Protein expression levels of STAT3, pSTAT3, and IL-17 in the different drug treatment groups were significantly decreased.

Conclusion: Jinwujiangu decoction inhibits the secretion of IL-6 and TGF- $\beta$ in RA-FLS, and intervenes to regulate gene expression of IL-23/IL-17 inflammation axis and suppress immune inflammation. The results of this study provide new evidence for the study of anti-inflammatory mechanism of TCM compound prescription.
\end{abstract}

Keywords: Jinwujiangu decoction, IL-17/IL-23, Fibroblast-like synoviocytes, Rheumatoid arthritis, Ethnomedicine

This is an Open Access article that uses a funding model which does not charge readers or their institutions for access and distributed under the terms of the Creative Commons Attribution License (http://creativecommons.org/licenses/by/4.0) and the Budapest Open Access Initiative (http://www.budapestopenaccessinitiative.org/read), which permit unrestricted use, distribution, and reproduction in any medium, provided the original work is properly credited.

Tropical Journal of Pharmaceutical Research is indexed by Science Citation Index (SciSearch), Scopus, International Pharmaceutical Abstract, Chemical Abstracts, Embase, Index Copernicus, EBSCO, African Index Medicus, JournalSeek, Journal Citation Reports/Science Edition, Directory of Open Access Journals (DOAJ), African Journal Online, Bioline International, Open-J-Gate and Pharmacy Abstracts

\section{INTRODUCTION}

Rheumatoid arthritis (RA) is an autoimmune disease, in which inflammatory synovitis and synovial joint erosion is the main reason for bone destruction and joint deformity [1]. Fibroblast-like synoviocytes (FLS) are stimulated to proliferate by the inflammatory process $[2,3]$. FLS secrete 
large amounts of inflammatory factors and mediate joint destruction in RA. Therefore, inhibiting the abnormal proliferation of FLS and preventing the release of inflammatory factors are crucial in the treatment of RA.

Recently, some reports have shown that the interleukin (IL)-17/IL-23 axis plays a key part in RA development $[4,5]$. IL-17 is an important proinflammatory cytokine in the pathogenesis of RA. It mainly exists in the synovial fluid of RA patients. Its secretion is closely related to anticyclic citrullinated peptide antibodies, $\lg \mathrm{M}, \lg \mathrm{A}$ and IgG. IL-17 is valuable as a marker to confirm active RA disease [6]. Additionally, IL-17 has a strong inflammatory effect and may play a critical role in aggravating joint destruction and synovial inflammation in RA. It stimulates stromal cells to secrete inflammatory factors such as IL-6, TNF- $\alpha$ and chemokines, $s$, and increase synovial inflammation in RA $[5,7]$.

IL-23 is a recently discovered cytokine that is expressed in a variety of cells, which is necessary for Th17 cell development and induces interferon (IFN) production [8]. In recent years, some studies have shown that IL-23 is a key cytokine in the pathogenesis of RA [9]. IL-23 gene-targeted mice were resistant to the development of osteoarthritis, so it may be a promoter of joint autoimmune inflammation.

The Miao medicine, Jinwujiangu decoction, has been used in the treatment of clinical experience of RA for a long time. It consists of Rhizoma cibotii, Periploca forrestii schltr, Caulis sinomenii, Homalomena occulta, Curcuma longa, Zaocys dhumnade, pseudo-ginseng, and licorice, with strong effects on kidney function, strengthening sinews, bone activation, expelling pathogenic wind, and tongluo. Previous studies showed that Jinwujiangu decoction could improve the clinical symptoms of joint pain and morning stiffness in RA patients [10]. It would reduce the protein activity of synovial cells and the secretion of IL17 and IL-23 in serum of CIA rats.

This study further investigated the effects of the Miao medicine, Jinwujiangu decoction, on the IL17/IL-23 axis in RA-FLS, to explore the antiinflammatory effects of Jinwujiangu decoction on RA - FLS.

\section{EXPERIMENTAL}

\section{Patients}

The samples were obtained from Guiyang Second Affiliated Hospital of Traditional Chinese Medicine and Guiyang Orthopaedic Hospital. All
RA patients and healthy controls according to the 1987 ACR classification criteria were enrolled and provided written informed consent [11].

\section{Isolation and culture of synoviocytes}

Synovial cells were obtained from patients with RA who underwent arthroplasty. The RA synovial tissue was placed in a culture dish to remove fat and blood clot, and the tissue was cleaned with PBS for three times. Then, the tissue was cut into $1 \mathrm{~mm}$ pieces and inoculated into $25 \mathrm{~mL}$ culture flask. At $37{ }^{\circ} \mathrm{C}$ and $5 \% \mathrm{CO}_{2}, 5 \mathrm{~mL}$ complete culture medium was added and cultured for 4 hours. To remove non-adherent lymphoid cells, the cell culture medium was changed after 3 days. They were passaged till monolayer cells spread to $70-80 \%$ of the bottom of the culture flask after enzymatic digestion with $0.25 \%$ trypsin. Cells at passages $3-5$ were used for the experiment [12-14].

\section{Experimental medicine}

Miao medicine, Jinwujiangu decoction, consists of Rhizoma cibotii $10 \mathrm{~g}$, Periploca forrestii schltr $10 \mathrm{~g}$, Caulis sinomenii $10 \mathrm{~g}$, Homalomena occulta $10 \mathrm{~g}$, Curcuma longa $10 \mathrm{~g}$, Zaocys dhumnade $10 \mathrm{~g}$, pseudo-ginseng $3 \mathrm{~g}$, and Radix paeoniae alba $15 \mathrm{~g}$. The herbal medicine was obtained from Guizhou Tongjitang pharmacy.

\section{Experimental groups and drug delivery}

The experiment was conducted with six groups, in which cells were treated respectively with low-, medium-, and high-dose Jinwujiangu decoction (0.06 $\mathrm{mg} / \mathrm{mL}, \quad 0.6 \mathrm{mg} / \mathrm{mL}, \quad 6.0 \mathrm{mg} / \mathrm{mL}$, respectively), leflunomide, tripterygium glycosides $(0.03 \mathrm{mg} / \mathrm{mL})$, and blank control. The cells were inoculated into sterile 96-well plates and cultured in a $37{ }^{\circ} \mathrm{C}$ incubator (Thermo Fisher, 3100) in an atmosphere of $5 \% \mathrm{CO}_{2}$. After the cells adhered, different concentrations of experimental drugs were added into 96-well plates and added such as volume DMEM to blank control group. At $37{ }^{\circ} \mathrm{C}$ and $5 \% \mathrm{CO}_{2}$, the 96 well plates were cultured for $24 \mathrm{~h}$. Then, cell supernatants were collected for subsequent experiments.

\section{3-[4,5-Dimethylthiazol-2-yl]-2,5 diphenyl tetrazolium bromide (MTT) assay}

Synoviocytes in the logarithmic phase were collected, $15 \%$ fetal bovine serum (GIBCO, 10099-133) was added to the cell suspension, and $6 \times 10^{4}$ cells were added to each well in 96well plates at $37^{\circ} \mathrm{C}$ incubator (Thermo Fisher, 3100 ) for $24 \mathrm{~h}$ in an atmosphere of $5 \% \mathrm{CO}_{2}$. 
Then, $200 \mu \mathrm{L}$ of different concentrations of Jinwujiangu decoction were added to each well of the 96-well plates. Each group had five repetitions. An equal volume of DMEM was added to the wells in the blank control group. At $37{ }^{\circ} \mathrm{C}$ and $5 \% \mathrm{CO}_{2}$, the 96-well plates were cultured for $24 \mathrm{~h}$. Subsequently, DMEM and MTT were added to each hole. After waiting for $4 \mathrm{~h}$, discard the supernatant and add formazan. The absorbancee at a wavelength of $490 \mathrm{~nm}$ was monitored by EnzymeSign while cell proliferation rate was measured based on the reference cells [12].

\section{Enzyme-linked immunosorbent assay (ELISA)}

The serum levels of IL- 6 , TGF- $\beta$, and IL-17 were determined by ELISA kits (Abcam, USA), according to the manufacturer's instructions.

\section{Real-time PCR}

RNA was prepared by RNA extraction kit (QiAGEN). mRNA was synthesized into the cDNA through the cDNA synthesis kit (Applied Biosystems, Japan). ABI Prism 7500 was used to quantify RT-PCR. Primers for IL-23R, IL-17R, RORa, RORyt, and STAT3 were purchased from Applied Biosystems. Each sample was processed in triplicate, and the experiment was repeated three times. All primers are presented in Table 1.

\section{Western blot assay}

Cells were inoculated on a $60 \mathrm{~mm}$ plate, cultured at room temperature for 24 hours. Then, the cells were collected and washed three times with PBS, and the precooled lysate was added to the cells. The solution were thoroughly mixed, placed on ice about 20 minutes, and centrifuged for 20 minutes. The protein was blocked overnight at $4^{\circ} \mathrm{C}$ with $5 \%$ skim milk without antibodies after being transferred to a membrane. After washing with TBSH three times, the membranes were incubated with anti-STAT3 (EPR787Y), antiSTAT3 (phospho Y705), and anti-IL17RD (ABCAM, UK), at a dilution of $1: 2000$, at $37^{\circ} \mathrm{C}$ for $1 \mathrm{~h}$. Then, protein bands were developed using ECL reagents, and images were acquired using the ChemiDoc Imaging system.

\section{Statistical analysis}

The data were expressed as mean \pm standard deviation (SD) and analyzed by one-way analysis of variance (ANOVA). The threshold was set to $p$ $<0.05$. SPSS 17 software was used for statistical analysis. Significance threshold was set at $p<$ 0.05 . SPSS 17.0 software was used for statistical analyses.

\section{RESULTS}

\section{Jinwujiangu decoction inhibited proliferation of RA synovial cells}

MTT testing results show that with increasing drug concentrations, the OD value gradually dropped, and the inhibition rate rose gradually (Figure 1). With drug concentrations in the $0.3-$ $0.6 \mathrm{mg} / \mathrm{mL}$ range, half-maximal inhibition of $\mathrm{RA}$ FLS proliferation was reached. At a concentration of $6.0 \mathrm{mg} / \mathrm{mL}$, the inhibition rate reached its peak value.

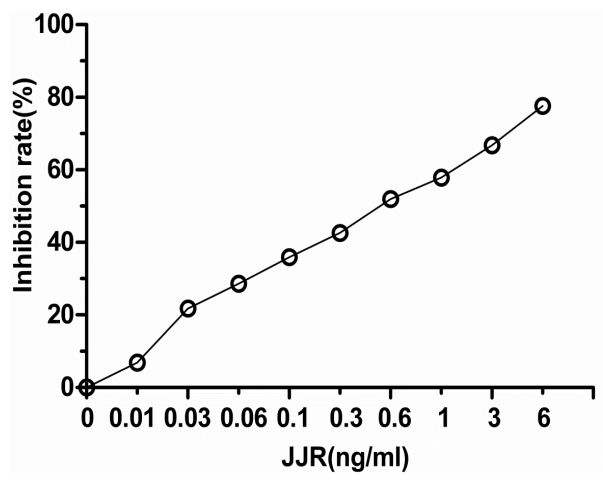

Figure 1: Inhibition of RA-FLS proliferation at different concentrations of Jinwujiangu decoction

\section{Effect of Jinwujiangu decoction on RA-FLS morphology}

When RA-FLS were treated with different drugs for $24 \mathrm{~h}$, cell morphological changes were investigated by reverse microscopy.

Table 1: Primer sequences

\begin{tabular}{lllc}
\hline Gene & Forward primer forward (5'-3') & Reverse primer (5'-3') & Size (bp) \\
\hline 3-actin & TCC TCC TGA GCG CAA GTA CTC T & GCT CAG TAA CAG TCC GCC TAG AA & 153 \\
IL-23R & TGC CTT GCA ATC TGA ACT TG & GAG CTC CCG GGA ATT CTT AC & 244 \\
IL-17R & CAGAAATGCCAGACACTCCA & CAGACGATGAGCAGGATGAC & 115 \\
STAT3 & TGG AGG AGA GAA TCG TGG AG & TTT GAC CAG CAA CCT GAC TTT & 149 \\
RORa & TCC CTA CTG TTC GTT CAC CA & CAG GTT TCC AGA TGC GAT TT & 102 \\
RORyt & GGC TGT GGG ACA AGT TCA GT & GTC GGA GAA GGT CAT GGT GT & 197 \\
\hline
\end{tabular}


Cells of the blank control group were arranged in close parallel swirls and were bright and shuttleshaped (Figure $2 \mathrm{~A}$ ). Compared with the blank control group, RA-FLS growth slowed with increased concentrations of Jinwujiangu decoction, and the swirling pattern disappeared. Cells became rounded and smaller and were spaced further apart. Cell adherence was decreased and the cells lost cytoplasm (Figure 2 $B$ to D). Compared with the tripterygium glycoside group (Figure $2 \mathrm{E}$ ), the medium-dose group did not show obvious differences. In the high-dose group, the cell morphology was obviously altered, the cells had very little cytoplasm, cellular size was reduced, and some cells underwent apoptosis. These changes were similar to those seen in the leflunomide group (Figure $2 \mathrm{~F}$ ). Cellular cytoplasm was lost, growth polarity disappeared, the cells became rounded and underwent apoptosis, and some cells were detached and floating in suspension.
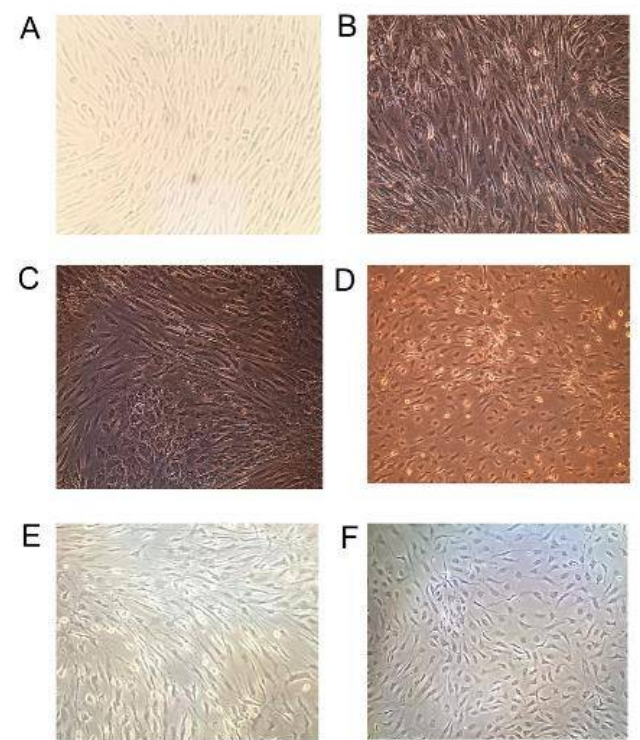

Figure 2: Effect of Jinwujiangu decoction on RA-FLS morphology. Note: A: Blank control group; B: Jinwujiangu decoction low doses group; C: Jinwujiangu decoction medium doses group; D: Jinwujiangu decoction high doses group; D: Tripterygium glycosides group; E: Leflunomide group)

\section{Effect of Jinwujiangu decoction on secretion of IL-6, TGF- $\beta$, and IL-17}

The results of ELISA (Figure 3 ) showed that the concentrations of IL- 6, TGF- $\beta$, and IL- 17 in cell culture supernatant were lower than those in blank control group $(p<0.01)$. Different concentrations of Jinwujiangu decoction resulted in decreased secretion of IL-6, TGF- $\beta$, and IL-17. The effect of high-dose treatment was more obvious than others $(p<0.01)$. Compared with the tripterygium glycosides group, the secretion levels of IL-6, TGF- $\beta$ and IL-17 in the high-dose group of Jinwujiangu Decoction decreased $(p<$ $0.05)$, while the secretion levels of IL-17 in the medium-dose group had no significant difference $(p<0.05)$. Compared with leflunomide group, the secretion levels of IL-6, TGF- $\beta$ and IL-17 in highdose Jinwujiangu decoction group were decreased $(p<0.01)$, but there was no significant difference in low-dose group and middle-dose group $(p>0.05)$.

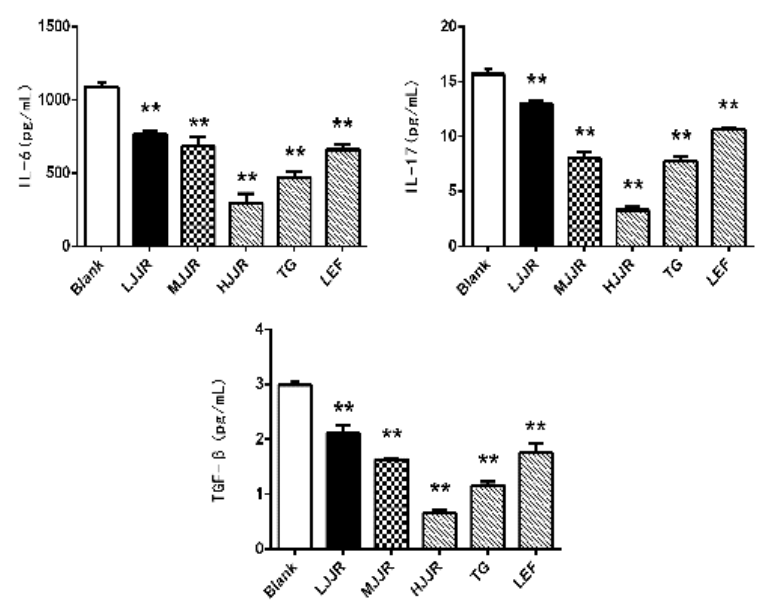

Figure 3: Effect of Jinwujiangu decoction on the secretion of IL- 6 , TGF- $\beta$, and IL- $17 ;{ }^{* *} p<0.01$

\section{Effect of Jinwujiangu decoction on expression of IL-23R, IL-17R, RORa, RORyt, and STAT3 mRNA}

The Figure 4 shown that the expression levels of IL-23R, IL-17R, RORa, RORyt, and STAT3 mRNA in RA-FLS were reduced following intervention with different drugs $(p<0.01)$. Compared with the tripterygium glycosides group, the expression levels of IL - 23R, IL - 17R, RORa, RORyt, and STAT3 mRNA in RA-FLS were lowest in the high-dose group ( $p<0.01$ ). Compared with the leflunomide group, the expression levels of IL-17R, RORyt, and STAT3 mRNA in RA-FLS in the low-dose group exhibited no obvious difference $(p>0.05)$. However, the decrease in expression of IL-23R, IL-17R, RORa, RORyt, and STAT3 mRNA in the high-dose group was less than in the leflunomide group $(p<0.01)$.

\section{Effect of Jinwujiangu decoction on protein activity of IL-17R, STAT3 and PSTAT3}

Western blot test results showed that the protein expression levels of STAT3, pSTAT3 and IL-17 in different drug treatment groups were significantly lower than those in blank control group $(p<0.01)$. Compared with the tripterygium glycosides group, the expression 
level of IL-17R protein in the middle dose group had no significant difference $(p>0.05)$. The expression levels of STAT3, pSTAT3 and IL-17R in high dose group were significantly decreased $(p<0.05)$. However, compared with leflunomide group, the expression levels of STAT3, pSTAT3 and IL-17R in high dose group were lower $(p<$ 0.01 ).
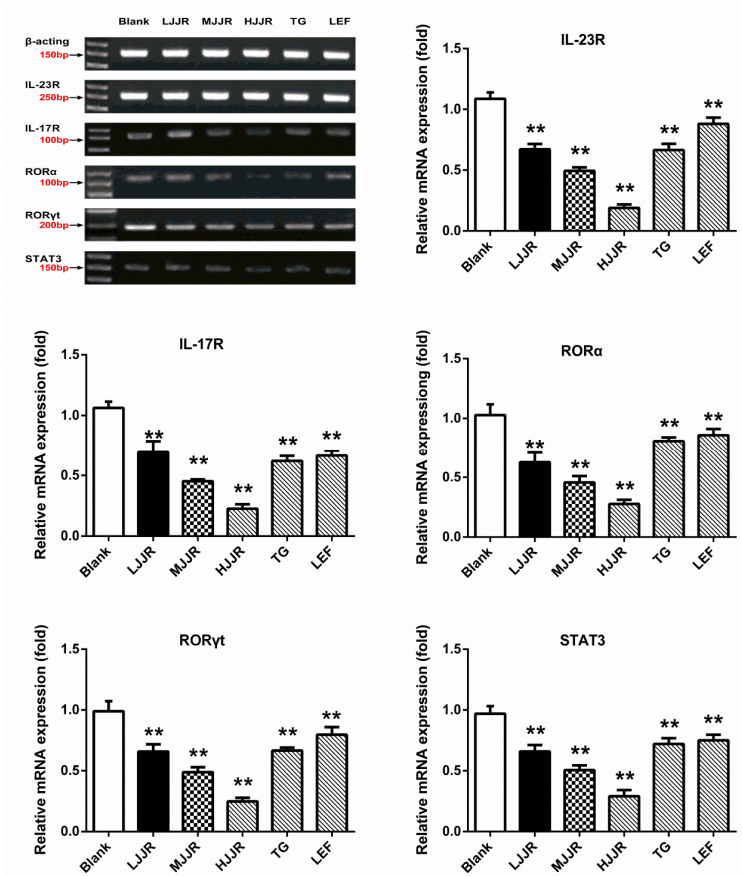

Figure 4: Expression of IL-23R, IL-17R, RORa, RORyt, and STAT3 mRNA in different groups; ${ }^{* *} p<$ 0.01
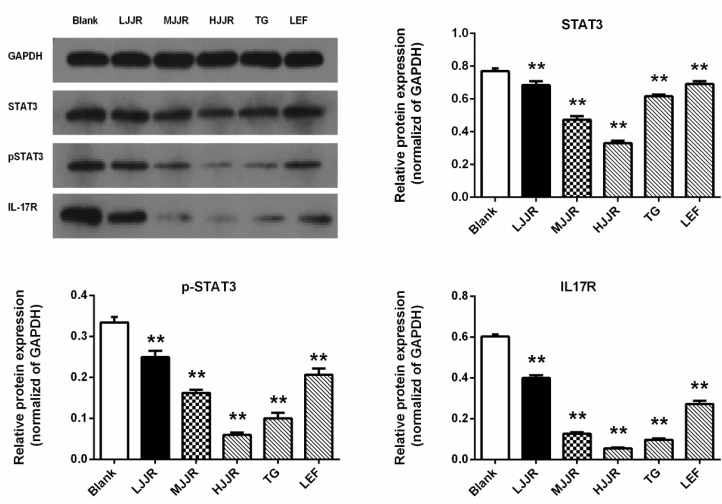

Figure 5: Protein expression levels of IL-17R, STAT3, and pSTAT3 in different groups. ${ }^{*} P<0.05 ;{ }^{* *} p<0.01$

\section{DISCUSSION}

RA is a systemic inflammatory disease. Most patients with RA will eventually have different degrees of joint dysfunction, which causes serious harm to human health. Some reports have shown that IL-17/IL-23 inflammatory axis has great significance for the improvement of joint synoviocytes in RA [15]. JAK2/STAT3 signaling pathway plays a bridge role in the transmission of IL-23/IL-17 inflammation axis. STAT3 plays a significant role in the differentiation process of Th17. Following the binding of IL-23 with its receptor, the JAK2/STAT3 signaling pathway was activated, causing the phosphorylation of STAT3 and promoting the expression of inflammatory mediators.

RORyt is a transcription factor for Th17 cells to secrete IL-17 and IL-23. Th17 differentiation is usually mediated by IL-17 and IL-23[16,17]. JAK2 is an essential factor in the phosphorylation process of STAT3, and the phosphorylation level of STAT3 is greatly reduced through the inhibiting activity of JAK2 [18]. Therefore, IL-23 activates JAK2/STAT3 pathway to upregulate the expression of RORyt. This study found that Jinwujiangu decoction could up-regulate the expression of IL-23R, STAT3, PSTAT3RO, and RORyt in RA-FLS. We speculate that Jinwujiangu decoction could weaken the bridge role of the JAK2/STAT3 genes in IL-23/IL-17 inflammation axis, and thereby inhibit the inflammatory effect of the IL-23/IL-17 axis. In addition, regulation of STAT3 is closely related to cell proliferation and apoptosis [19]. Studies have confirmed that STAT3 directly inhibits the proliferation of RA-FLS [20]. This study shows that compared with the blank control group, different concentrations of Jinwujiangu decoction have an inhibitory effect on RA-FLS proliferation, which could upregulate the gene and protein expression levels of STAT3.

RORa and RORyt are members of a family of steroid hormone receptors. RORyt is an important transcriptional regulator that mediates the function of IL-23/IL-17 inflammation axis and promotes the secretion of IL-17 [21]. Among them, RORyt is more effective in promoting the secretion of IL-17, and both RORa and RORyt in combination are more effective than either in isolation. In the absence of both RORa and RORyt, IL-23/IL-17 axis would be unable to produce an inflammatory effect. This study found that Jinwujiangu decoction could up-regulate the expression of RORa and RORyt in RA patients, enhance the inhibitory effect of IL-17 secretion, and prevent the persistent inflammation of RA.In normal circumstances, proinflammatory and antiinflammatory cytokines are balanced to maintain homeostasis in the body. They form a complex cell network and mediate a variety of important physiological functions of the body. Increase in immune dysfunction and proinflammatory cytokines is one of the important mechanisms of 
RA progression. IL-6 is a proinflammatory factor that has a variety of biological activities. It regulates the activity of other cytokines and of multiple signaling pathways [22]. Transforming growth factor- $\beta$ (TGF- $\beta$ ) is a multifunctional cell regulatory factor, and has a dual role in RA. The binding of TGF- $\beta$ with TGF- $\beta$ receptors on the surface of osteoblast precursor cells promotes the differentiation and expression of osteoblasts $[23,24]$.

This study suggests that Jinwujiangu decoction can inhibit the secretion of IL- 6 and TGF- $\beta$ in RAFLS, interfere with the gene expression of IL23/IL-17 inflammation axis and inhibit immune inflammation. However, due to the different biological effects of IL- 6 and TGF- $\beta$, the effects of Jinwujiangu decoction on RA pannus hyperplasia and bone destruction need to be further study. We will further understand the detailed mechanism of Jinwujiangu decoction in the treatment of rheumatoid arthritis.

\section{CONCLUSION}

In this study, the in vitro culture models of different concentrations of Jinwu Jiangu Decoction for RA-FLS were successfully established, and the mechanism of Jinwu Jiangu Decoction on the inflammation axis of RA IL23/IL-17 was discussed. They suggested that Jinwu Jiangu Decoction plays an important role in immunopathology of rheumatoid arthritis. The results of this study open up new avenues for the development of ethnomedicinal therapeutics for RA.

\section{DECLARATIONS}

\section{Acknowledgement}

This work was supported by Natural Science Foundation of China which entitled, 'Discuss the Mechanism of Miao Medicine Jinwu Jiangu Capsule to Rheumatoid Arthritis Synovial Cell Autophagy and Apoptosis Based on mTOR Signal' (grant no. 81760907); Guizhou province high-level innovative talent training program by Guizhou Provincial Department of Science and Technology (grant no. [2016]5650).

\section{Conflict of Interest}

No conflict of interest associated with this work.

\section{Contribution of Authors}

We declare that this work was done by the authors named in this article and all liabilities pertaining to claims relating to the content of this article will be borne by the authors. Xueming Yao and Wukai Ma designed all the experiments and revised the paper. Rong Li, Qiaoyi Ning, Ying Huang, Fang Tang, and Hui Xu performed the experiments. Daoming Lu, Jiang Liang, and Yang An wrote the paper.

\section{REFERENCES}

1. Salt E, Crofford L. Rheumatoid arthritis: new treatments, better outcomes. Nurse Practitioner. 2012; 37(11): 2223.

2. Bartok B, Firestein GS. Fibroblast-like synoviocytes: key effector cells in rheumatoid arthritis. Immunol Rev. 2010; 233(1): 233-255.

3. Bottini $N$, Firestein GS. Duality of fibroblast-like synoviocytes in $R A$ : passive responders and imprinted aggressors. Nat Rev Rheumatol. 2013; 9(1): 24-33.

4. Al-Saadany HM, Hussein MS, Gaber RA, Zaytoun HA. Th-17 cells and serum IL-17 in rheumatoid arthritis patients: Correlation with disease activity and severity. Egyp Rheumatol. 2016; 38(1): 1-7.

5. Paradowskagorycka A, Grzybowskakowalczyk A, Wojteckalukasik E, Maslinski $S$. IL-23 in the pathogenesis of rheumatoid arthritis. Scandinavian $J$ Immunol. 2010; 71(3): 134-145.

6. Kim KW, Kim HR, Kim BM, Cho ML, Lee SH. Th17 cytokines regulate osteoclastogenesis in rheumatoid arthritis. Am J Pathol. 2015; 185(11): 3011-3024.

7. Azizi G, Jadidi-Niaragh F, Mirshafiey A. Th17 Cells in Immunopathogenesis and treatment of rheumatoid arthritis. Int J Rheum Diseases. 2013; 16(3): 243-253.

8. Frucht DM. IL-23: a cytokine that acts on memory $T$ cells. Sci Stke. 2002; 2002(114): pe1.

9. Kobezda T, Ghasseminejad S, Mikecz K, Glant TT, Szekanecz Z. Of mice and men: how animal models advance our understanding of T-cell function in RA. Nat Rev Rheumatol. 2014; 10(3): 160.

10. Huang H, Cao YP, Yao XM, Ma WK. Clinical study of Miao Nationality Medicine Jinwujiangu Decoction and $D M A R D s$ in the treatment of rheumatoid arthritis. Rheum Arthr. 2012.

11. Arnett FC, Edworthy SM, Bloch DA, Mcshane DJ, Fries $J F$, Cooper NS, Healey LA, Kaplan SR, Liang $M H$, Luthra HS. The American Rheumatism Association 1987 revised criteria for the classification of rheumatoid arthritis. Rheum Arthr. 1988; 31(3): 315-324.

12. Chen $X Y$, Li J, Cheng WM, Jiang $H$, Xie XF, Hu R. Effect of total flavonoids of Chrysanthemum indicum on the apoptosis of synoviocytes in joint of adjuvant arthritis rats. Am J Chi Med. 2008; 36(04): 695-704.

13. Chen $Q Q$, Xiao P, Liu HX, Zhang YF, Stomatology DO. Cultivation of condylar chondrocytes and its biological characteristics in rats. J Chi Pract Dia Ther. 2017.

14. Iwamoto $T$, Okamoto $H$, Shu K, Ikari K, Toyama $Y$, Tomatsu T, Kamatani $N$, Momohara $S$. A role of monocyte chemoattractant protein-4 (MCP-4)/CCL13 
from chondrocytes in rheumatoid arthritis. Febs J. 2010; 274(18): 4904-4912.

15. Kim HR, Cho ML, Kim KW, Juhn JY, Hwang SY, Yoon $\mathrm{CH}$, Park SH, Lee SH, Kim HY. Up-regulation of IL$23 p 19$ expression in rheumatoid arthritis synovial fibroblasts by IL-17 through PI3-kinase-, NF-KB- and p38 MAPK-dependent signalling pathways. Rheumatology. 2007; 46(1): 57-64.

16. Lubberts $E$. The IL-23-IL-17 axis in inflammatory arthritis. Nat Rev Rheumatol. 2015; 11(7): 415-429.

17. Hirahara $K$, Ghoreschi $K$, Laurence A, Yang XP, Kanno $Y$, O'Shea JJ. Signal transduction pathways and transcriptional regulation in Th17 cell differentiation. Cytokine Growth F R. 2010; 21(6): 425-434.

18. Yeremenko N, Paramarta JE, Baeten D. The interleukin23/interleukin-17 immune axis as a promising new target in the treatment of spondyloarthritis. Cur Opin Rheumatol. 2014; 26(4): 361-370.

19. Saleh A, Shan L, Halayko AJ, Kung S, Gounni AS. Critical role for STAT3 in IL-17A-mediated CCL11 expression in human airway smooth muscle cells. J Immunol. 2009; 182(6): 3357-3365.

20. Leemanneill RJ, Seethala RR, Singh SV, Freilino $M L$, Bednash JS, Thomas SM, Panahandeh MC, Gooding WE, Joyce SC, Lingen MW. Inhibition of EGFR-STAT3 signaling with erlotinib prevents carcinogenesis in a chemically-induced mouse model of oral squamous cell carcinoma. Cancer Prevent Res. 2011; 4(2): 230.

21. Krause A, Scaletta N, Ji JD, Ivashkiv LB. Rheumatoid Arthritis Synoviocyte Survival Is Dependent on Stat3. J Immunol. 2002; 169(11): 6610.

22. Szekanecz Z, Koch AE. Targeting Angiogenesis in Rheumatoid Arthritis. Curr Rheumatol Rev. 2008; 4(4): - .

23. Stockinger B, Veldhoen M, Martin B. Th17 T cells: linking innate and adaptive immunity. Sem Immunol. 2007; 19(6): 353-361.

24. Palioto DB, Rodrigues TL, Marchesan JT, Beloti MM, Oliveira PTD, Rosa AL. Effects of enamel matrix derivative and transforming growth factor- $\beta 1$ on human osteoblastic cells. Head Face Med. 2011; 7(1): 13-13. 